\title{
Shape Segmentation and Matching with Flow Discretization
}

\author{
Tamal K. Dey \\ Dept. of CSE \\ The Ohio State U. \\ Columbus, Ohio 43081 \\ USA
}

Joachim Giesen

Theoretische Informatik

ETH Zürich

CH-8092 Zürich

Switzerland

\author{
Samrat Goswami
}

\author{
Dept. of CSE \\ The Ohio State U. \\ Columbus, Ohio 43081 \\ USA
}

\begin{abstract}
Geometric shapes are identified with their features. For computational purposes a concrete mathematical definition of features is required. In this paper we use a topological approach, namely dynamical systems, to define features of shapes. To exploit this definition algorithmically we assume that a point sample of the shape boundary is given as input from which features of the shape have to be approximated. We translate our definition of features to the discrete domain while mimicking the set-up developed for the continuous shapes. The outcome of this approach is a clean mathematical definition of features that are efficiently computable with combinatorial algorithms. Experimental results show that our algorithms segment shapes in two and three dimensions into so-called features quite effectively. Further, we develop a shape matching algorithm that takes advantage of our robust feature segmentation step. Performance of this algorithm is exhibited with experimental results.
\end{abstract}

\section{Introduction}

The features of a shape are its specific identifiable subsets. Although this high level characterization of features is assumed routinely, more concrete and mathematical definitions are required for computational purposes. Many applications including object recognition, classification, matching, tracking need to solve the problem of segmenting a shape into its salient features, see for example $[9,10,20,28,34$, 41]. Most of these applications need an appropriate definition of features that are computable. In the computational domains, the shapes are often represented with discrete means that approximate them. Consequently, a consistent definition of features in the discrete domain is needed to compute them reliably.

Different geometric and topological structures such as shock graphs [35, 37], medial axes [26], Reeb graphs [20], watersheds [29] and others [24, 27] have been proposed in the past for shape segmentation. Two notable topological approaches related to shape features are level sets method [36] and the topological persistence [16]. The level sets method use numerical techniques to compute features whereas we rely more on combinatorial means. This makes computations faster and more robust against numerical 
errors. Topological persistence method works with homological algebra to compute a signature of the shape that respects its features, but do not address the segmentation issue.

In this paper we use a topological approach, namely dynamical systems, to define features of shapes. This approach has been studied in the context of surface reconstruction recently $[15,18]$. We assume that a point sample of the shapes is given as input from which features of the shape have to be approximated. We translate our definition of features to this discrete domain while mimicking the set-up that we develop in the continuous case. The outcome of this approach is a clean mathematical definition of features that are computable with combinatorial algorithms. For shapes in the plane we compute them exactly whereas we approximate them for shapes embedded in $\mathbb{R}^{3}$ mimicking the two dimensional algorithm. Our experimental results show that our algorithms segment shapes in two and three dimensions into so-called features quite effectively.

We apply our feature segmentation technique to the shape matching problem, which seeks a similarity measure between two shapes. An usual approach in shape matching is to compute a signature of a shape and then comparing it with the signature of the other shape. Different quantities such as curvature distribution [3, 38], wavelet coefficients [22], Fourier descriptors [2], geometric statistics [4, 33, 39], spin image [23] and shape distribution [25, 30] have been suggested for shape signatures; see survey articles $[1,7,28,40]$ for more details. Another prevalent approach is to segment a shape into its salient features and then match the shapes based on the features and their spatial relationships $[5,6,8,19]$. These feature based approaches depend mainly on the quality of the feature detection step. We give a shape matching algorithm that takes the advantage of our robust feature segmentation step. Each significant feature segment is represented with a weighted point where the weight is the volume of the segment. Then, the shape matching problem boils down to matching two small weighted point sets instead of matching large point sets derived from the boundary of the shapes [21]. We carry out these steps so that the entire matching process remains invariant to rotation, translation, mirroring and scaling.

\section{Flow and critical points}

In this section we outline a theory of the flow induced by a shape. Later we will use this theory to define and compute features of shapes. Here we will develop the theory in a more general setting by considering compact hypersurfaces of the $d$-dimensional Euclidean space $\mathbb{R}^{d}$, i.e., we consider compact, $(d-1)$-dimensional submanifolds of $\mathbb{R}^{d}$.

Shape. Let $\Sigma$ be a compact $(d-1)$-manifold without boundary embedded in $\mathbb{R}^{d}$. The closure of the bounded component of $\mathbb{R}^{d} \backslash \Sigma$ is the shape interior denoted $\operatorname{In}(\Sigma)$. The shape exterior denoted $\operatorname{Ex}(\Sigma)$ is the closure of the unbounded component of $\mathbb{R}^{d} \backslash \Sigma$. The segmentation method partitions $\operatorname{In}(\Sigma)$ and $\operatorname{Ex}(\Sigma)$ simultaneously. In what follows, by shape we will mean both $\operatorname{In}(\Sigma)$ and $\operatorname{Ex}(\Sigma)$ each of which is bounded by $\Sigma$.

Height function. The manifold $\Sigma$ can be used to define a distance function $h: \mathbb{R}^{d} \rightarrow \mathbb{R}$ as

$$
h(x)=\min _{p \in \Sigma}\|p-x\|^{2} \text { for all } x \in \mathbb{R}^{d} .
$$

Anchor set. Associated with the distance function, we define an anchor set for each point $x \in \mathbb{R}^{d}$ as

$$
A(x)=\operatorname{argmin}_{p \in \Sigma}\|p-x\| .
$$


Basically, $A(x)$ is the set of closest point(s) to $x$ in $\Sigma$; see Figure 1 . Note that $A(x)$ can contain even a continuum of points.

We would like to define a unit vector field $v: \mathbb{R}^{d} \rightarrow \mathbb{R}^{d}$ that assigns to every point $x \in \mathbb{R}^{d}$ the direction in which the distance function increases the most. If $h$ is smooth at $x$ then $v(x)$ coincides with the normalized gradient $\nabla h(x) /\|\nabla h(x)\|$. In our case $h$ is not smooth everywhere. So, we have to be careful to define $v(x)$ at any non-smooth point $x$. Instead of smooth and non-smooth points we will talk about regular and critical points in the following. Critical points are either local extrema or saddle points of the distance function. We use a generalized theory of critical points, see for example [31], to derive the following definition.

Regular and critical point. For every point $x \in \mathbb{R}^{d}$ let $H(x)$ be the convex hull of $A(x)$, i.e. the convex hull of the points on $\Sigma$ that are closest to $x$. We call $x$ a critical point of $h$ if $x \in H(x)$. Otherwise we call $x$ a regular point.

The following definition turns out to be very helpful in the subsequent discussion. It allows us to characterize the direction of steepest ascent of the distance function $h$ at every point $x \in \mathbb{R}^{d}$.

Driver. For any point $x \in \mathbb{R}^{d}$ let $d(x)$ be the point in $H(x)$ closest to $x$. Note that this point is unique since $H(x)$ is convex. We call $d(x)$ the driver of $x$.

Lemma 1 For any regular point $x \in \mathbb{R}^{d}$ let $d(x)$ be the driver of $x$. The steepest ascent of the distance function $h$ at $x$ is in the direction of $x-d(x)$.

PROOF. Note that $h$ is continuous though it is not smooth.

Our assumption that $x$ is a regular point, i.e. $x \notin H(x)$, implies that the vector $x-d(x)$ is non-zero. To prove our claim we want to show first that $h$ is non-increasing in all directions that make an angle of $\pi / 2$ or larger with $x-d(x)$.

By definition all points in $A(x)$ have the same distance $d$ from $x$ and $|A(x)| \geq 1$. For any $p \in A(x)$ let $W_{p}$ be the unique closed halfspace that has $x$ in its boundary and contains the ball of radius $\|x-d(x)\|$ centered at $p$. Obviously, the distance function $h$ at $x$ is decreasing in all directions that point into the interior of $W_{p}$ for all $p \in A(x)$. Hence by the continuity of $h$ it is non-increasing in all directions that point into $W_{p}$. Let

$$
W=\bigcup_{p \in A(x)} W_{p} .
$$

By construction all directions at $x$ that make an angle of $\pi / 2$ or larger with $x-d(x)$ point into $W$. Thus $h$ is non-increasing in all those directions. This implies that $h$ can only be increasing in some direction $v$ if $v \cdot(x-d(x))^{T}>0$. One can decompose $v$ as $v=v_{1}+v_{2}$ where $v_{1}$ is along $x-d(x)$ and $v_{2}$ is orthogonal to it. By our previous argument, $h$ is non-increasing along $v_{2}$. It follows that the increase of $h$ is maximized if its component along $v_{2}$ vanishes, i.e., $h$ has its steepest ascent at $x$ in the direction $x-d(x)$. 


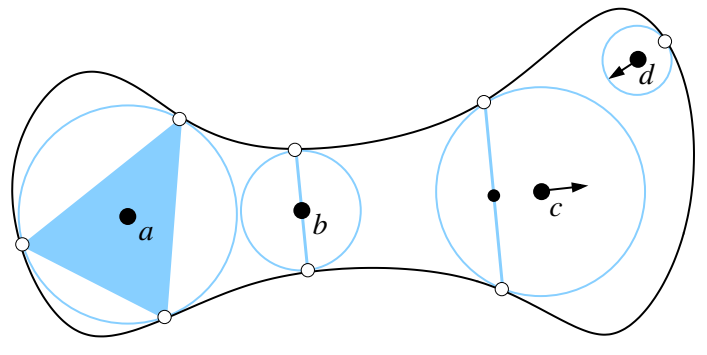

Figure 1: In this example $\Sigma$ is a curve embedded in $\mathbb{R}^{2}$. The sets $A(x)$ are shown with hollow circles for four points $x=a, b, c, d \in \mathbb{R}^{2}$. The convex hulls of $A(x)$ are light shaded. The driver of the point $c$ is the smaller black circle. The driver of the point $d$ is the single point in $A(d)$. The points $a$ and $b$ are critical since they are contained in $H(a)$ and $H(b)$, respectively. The points $c$ and $d$ are regular. The directions of steepest ascent of the distance function at $c$ and $d$ are indicated by arrows.

We are now going to use the direction of steepest ascent to define a flow on $\mathbb{R}^{d}$, i.e. a dynamical system on $\mathbb{R}^{d}$.

Induced flow. Define a vector field $v: \mathbb{R}^{d} \rightarrow \mathbb{R}^{d}$ by setting

$$
v(x)=\frac{x-d(x)}{\|x-d(x)\|} \text { if } x \neq d(x) \text { and } 0 \text { otherwise. }
$$

Note, that the vector field vanishes exactly at the critical points since $x \neq d(x)$ holds for all regular points. The flow induced by the vector field $v$ is a function $\phi:[0, \infty) \times \mathbb{R}^{d} \rightarrow \mathbb{R}^{d}$ such that the right derivative at every point $x \in \mathbb{R}^{d}$ satisfies the following equation:

$$
\lim _{t \downarrow t_{0}} \frac{\phi(t, x)-\phi\left(t_{0}, x\right)}{t-t_{0}}=v\left(\phi\left(t_{0}, x\right)\right)
$$

Orbits and fixed points. Given $x \in \mathbb{R}^{d}$ and an induced flow $\phi$, the curve $\phi_{x}:[0, \infty) \rightarrow \mathbb{R}^{d}, t \mapsto$ $\phi(t, x)$ is called the orbit of $x$. A point $x \in \mathbb{R}^{d}$ is called a fixed point of $\phi$ if $\phi(t, x)=x$ for all $t \geq 0$.

Basically, the orbit of a point is the curve it will follow if it were let move along the flow.

Observation 1 The fixed points of $\phi$ are the critical points of the distance function $h$.

Because of this observation we refer to a fixed point of $\phi$ as a minimum, saddle or maximum if the corresponding critical point of the distance function is a minimum, saddle or maximum, respectively.

Stable manifold. The stable manifold $S(x)$ of a critical point $x$ is the set of all points that flow into $x$, i.e.

$$
S(x)=\left\{y \in \mathbb{R}^{d}: \lim _{t \rightarrow \infty} \phi_{y}(t)=x\right\}
$$


The stable manifolds of all critical points partition $\mathbb{R}^{d}$, i.e.

$$
\mathbb{R}^{d}=\bigcup_{\text {critical points } x} S(x)
$$

if we add a critical point at infinity, and $S(x) \cap S(y)=\emptyset$ for any two different critical points $x$ and $y$.

\section{Discretization}

To deal with continuous shapes algorithmically we discretize their boundaries. Here discretization means taking a finite point sample $P$ of the shape boundary $\Sigma \subset \mathbb{R}^{d}$. That is, we replace $\Sigma$ by a finite subset of $\Sigma$. The sample $P$ induces another vector field which resembles the vector field induced by $\Sigma$ provided $P$ is sufficiently dense in $\Sigma$. At the moment we cannot quantify this resemblance in terms of a sampling density, but it is quite intuitive that, for example, the prominent local maxima of both vector fields are very similar. The vector field induced by $P$ is intimately linked with the Voronoi and the Delaunay diagram of $P$. Moreover, the stable manifolds corresponding to the flow induced by this vector field are efficiently computable in dimensions two and three.

Let us first summarize the definitions of Voronoi and Delaunay diagrams before we show how the concepts we introduced in the last section can be specialized to the case of finite point sets.

Voronoi diagram. Let $P$ be a finite set of points in $\mathbb{R}^{d}$. The Voronoi cell of $p \in P$ is given as

$$
\left.V_{p}=\left\{x \in \mathbb{R}^{d}: \forall q \in P \backslash\{p\},\|x-p\| \leq\|x-q\|\right)\right\} .
$$

The sets $V_{p}$ are convex polyhedra or empty since the set of points that have the same distance from two points in $P$ forms a hyperplane. Closed facets shared by $k, 2 \leq k \leq d$, Voronoi cells are called $(d-k+1)$-dimensional Voronoi facets and points shared by $d+1$ or more Voronoi cells are called Voronoi vertices. The term Voronoi object denotes either a Voronoi cell, facet or vertex. The Voronoi diagram $V_{P}$ of $P$ is the collection of all Voronoi objects. It defines a cell decomposition of $\mathbb{R}^{d}$.

Delaunay diagram. The Delaunay diagram $D_{P}$ of a set of points $P$ is dual to the Voronoi diagram of $P$. The convex hull of $d+1$ or more points in $P$ defines a Delaunay cell if the intersection of the corresponding Voronoi cells is not empty and there exists no superset of points in $P$ with the same property. Analogously, the convex hull of $k \leq d$ points defines a $(k-1)$-dimensional Delaunay face if the intersection of their corresponding Voronoi cells is not empty. Every point in $P$ is called a Delaunay vertex. The term Delaunay object denotes either a Delaunay cell, face or vertex. The Delaunay diagram $D_{P}$ defines a decomposition of the convex hull of all points in $P$. This decomposition is a triangulation if the points are in general position.

The dual Delaunay object of a given Voronoi object is the convex hull of all points in $P$ whose Voronoi cells contain the Voronoi object.

We always refer to the interior and to the boundary of Voronoi/Delaunay objects with respect to their dimension, e.g. the interior of a Delaunay edge contains all points in this edge besides the endpoints. The interior of a vertex and its boundary are the vertex itself. Furthermore, unless stated differently, 

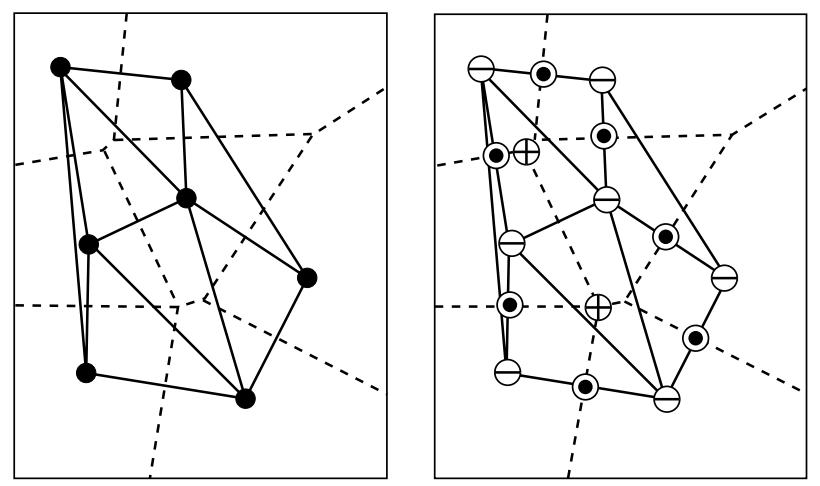

Figure 2: On the left: The Voronoi diagram (dashed lines) and the Delaunay triangulation (solid lines) of seven points in $\mathbb{R}^{2}$. On the right: The critical points (maxima $\oplus$, saddle points $\odot$ and minima $\ominus$ ) of the distance function induced by the seven points.

we always assume general position, i.e., no three points are collinear, no four points are cocircular or coplanar, and no five points are cospherical.

Now consider the distance function $h$ as in the previous section but replacing $\Sigma$ with its discrete sample $P$. Note that we are using the same definition of critical points for $h$ as we did in the continuous case. The following lemma is known, see for example $[15,18]$.

Lemma 2 Let $P$ be a finite set of points such that the Voronoi and their dual Delaunay objects intersect in their interiors if they intersect at all. Then the critical points of the distance function $h$ are the intersection points of Voronoi objects $V$ and their dual Delaunay object $\sigma$.

This characterization of critical points can be used to assign a meaningful index to critical points, namely, the index of a critical point is the dimension of the Delaunay object used in the above characterization. Minima always have index 0 and maxima always have index $d$.

The driver of a point in $\mathbb{R}^{d}$ can now also be described in terms of Voronoi and Delaunay objects, because the anchor hulls now are always Delaunay objects.

Observation 2 Given $x \in \mathbb{R}^{d}$. Let $V$ be the lowest dimensional Voronoi object in the Voronoi diagram of $P$ that contains $x$ and let $\sigma$ be the dual Delaunay object of $V$. The driver of $x$ is the point in $\sigma$ closest to $x$.

We have a much more explicit characterization of the flow induced by a finite point set than in the general case.

Observation 3 The flow $\phi$ induced by a finite point set $P$ is given as follows: For all critical points $x$ of the distance function associated with $P$ we set

$$
\phi(t, x)=x, t \in[0, \infty)
$$

Otherwise let $d(x)$ be the driver of $x$ and $R$ be the ray originating at $x$ and shooting in the direction $v(x)=(x-d(x)) /\|x-d(x)\|$. Let $z$ be the first point on $R$ whose driver is different from $d(x)$. Note 
that such a $z$ need not exist in $\mathbb{R}^{d}$ if $x$ is contained in an unbounded Voronoi object. In this case let $z$ be the point at infinity in the direction of $R$. We set

$$
\phi(t, x)=x+t \cdot v(x), t \in[0,\|z-x\|) .
$$

For $t \geq\|z-x\|$ the flow is given as

$$
\begin{aligned}
\phi(t, x) & =\phi(t-\|z-x\|+\|z-x\|, x) \\
& =\phi(t-\|z-x\|, \phi(\|z-x\|, x)) .
\end{aligned}
$$

It is not completely obvious, but it can be shown that this flow is well defined. It is also easy to see that the orbits of $\phi$ are piecewise linear curves that are linear in Voronoi objects. See Figure 4 for some examples of orbits.

Under some mild non-degeneracy condition the stable manifolds of the critical points have a nice recursive structure. A stable manifold of index $k, 0 \leq k \leq d$, has dimension $k$ and its boundary consists of stable manifolds of index $k-1$ critical points.

In $\mathbb{R}^{2}$ the stable manifolds of index 1 critical points, i.e. saddle points, are exactly the Delaunay edges whose circumcircle is empty. These edges also called Gabriel edges form the Gabriel graph of the point set $P$. The Gabriel graph is efficiently computable. The recursive structure of the stable manifolds now tells us that the stable manifolds of the maxima, i.e. index 2 critical points, are exactly the compact regions of the Gabriel graph. That is, the stable manifolds of maxima (index 2 critical points) are given as a union of Delaunay triangles.
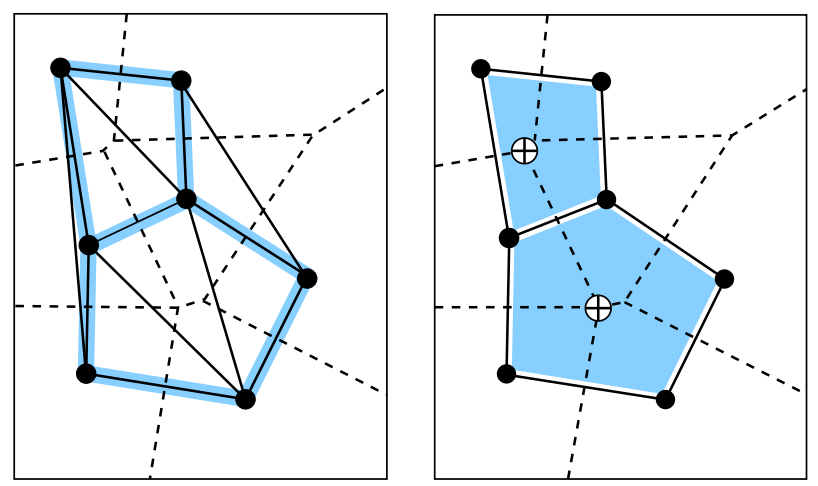

Figure 3: On the left: The edges of the Gabriel graph of the seven points from Figure 1 are highlighted. On the right: The stable manifolds of the maxima $\oplus$ of the flow induced by the seven points.

The stable manifolds of flows induced by finite point sets in $\mathbb{R}^{3}$ can also be computed efficiently, see [18]. But already in $\mathbb{R}^{3}$ the stable manifolds of index 2 saddle points and maxima are not subcomplexes of the three dimensional Delaunay triangulation. This is why we choose to bypass the exact computations of the stable manifolds and instead approximate them with sub-complexes of the Delaunay triangulation. The approximation is more efficient in size and computational cost than the exact one. 


\section{Approximating stable manifolds}

Our goal is to decompose a two or three dimensional shape into disjoint segments that respect the 'features' of the shape. In our first attempt to define features we resort to stable manifolds of maxima. So, we define a feature to be the closed stable manifold $F(x)$ of a maximum $x$,

$$
F(x)=\operatorname{closure}(S(x)) \text {. }
$$

Figure 5(a) shows the segmentation of a shape in $\mathbb{R}^{2}$ with this definition of features. We can translate this definition to the discrete setting immediately as we have mimicked all concepts in the continuous case to the discrete setting. Figure 5(b) shows this segmentation.

From a point sample $P$ of a shape boundary $\Sigma$ we would like to compute $F(x)$ for all maxima $x$. These maxima are a subset of the Voronoi vertices in $V_{P}$. For computing the feature segmentation it is sufficient to compute the boundary of all such $F(x)$. As we observed earlier this boundary is partitioned by the stable manifolds of critical points of lower indices. In $\mathbb{R}^{2}$ this means that Gabriel edges separate the features.

We also want to separate the features in $\mathbb{R}^{3}$ by a subset of the Delaunay triangles. That is, we want to approximate the boundary of the stable manifolds of maxima by Delaunay triangles. These boundaries consist of stable manifolds of critical points of index 1 and 2. The closures of the stable manifolds of index 1 critical points are again exactly the Gabriel edges. By Lemma 2 each critical point of index 2 lies in a Delaunay triangle which we call a saddle triangle. The stable manifolds of the index 2 critical points may not be contained only in the saddle triangles. This makes computing the boundary of the stable manifolds of maxima harder in $\mathbb{R}^{3}$. Although it can be computed exactly, we propose an alternative method that approximates this boundary using only Delaunay triangles. We derive this method by generalizing a simple algorithm that computes the closed stable manifolds for maxima in $\mathbb{R}^{2}$ exactly.

In $\mathbb{R}^{2}$ we can compute the closed stable manifold $F(x)$ of a maximum $x$ by exploring out from the Delaunay triangle containing $x$. To explain the algorithm we define a flow relation among Delaunay triangles which was proposed by Edelsbrunner et al. [17] for computing pockets in molecules.

Flow relation in $\mathbb{R}^{2}$. Let $\sigma_{1}, \sigma_{2}$ be two triangles that share an edge $e$. We say $\sigma_{1}<\sigma_{2}$ if $\sigma_{1}$ and its dual Voronoi vertex lie on the opposite sides of the supporting line of $e$.

Observation 4 Let $\sigma_{1}$ and $\sigma_{2}$ be two triangles sharing an edge e where $\sigma_{1}<\sigma_{2}$. Then the flow on the dual Voronoi edge $v_{1} v_{2}$ of e is directed from $v_{1}$ to $v_{2}$ where $v_{i}$ is the dual Voronoi vertex of $\sigma_{i}$.

It is obvious from the definition that the transitive closure $<^{*}$ of $<$ is acyclic [17]. If $\sigma_{1}<\sigma_{2}$, then the radius of the circumcircle of $\sigma_{2}$ is larger than the radius of the circumcircle of $\sigma_{1}$. So, in a chain of triangles related by $<$, the circumradii of the triangles can never decrease, thus making it impossible for $<^{*}$ to be cyclic. This means that, for each triangle $\sigma^{\prime}$, there is a triangle $\sigma$ containing a maximum $x$ such that $\sigma^{\prime}<^{*} \sigma$. We will say that $\sigma^{\prime}$ flows into $\sigma$.

The following lemma holds in $\mathbb{R}^{2}$.

Lemma 3 Let $\sigma$ be a triangle containing a maximum $x$. We have $F(x)=\bigcup_{\sigma^{\prime}<{ }^{*} \sigma} \sigma^{\prime}$. 
The algorithm, originally proposed by Edelsbrunner et al. [17], for computing the closed stable manifold $F(x)$ follows immediately from the above lemma. Initially $F(x)$ is set to the triangle $\sigma$ that contains $x$. At any generic step of this exploration, let $e$ be a Delaunay edge that lies on the boundary of $F(x)$ computed so far. Let $\sigma_{1}$ and $\sigma_{2}$ be two triangles that share $e$ where $\sigma_{1}$ is outside $F(x)$. If $\sigma_{1}<\sigma_{2}$ we update $F(x)$ as $F(x):=F(x) \cup \sigma_{1}$. This process continues till we cannot include any more triangle into $F(x)$.
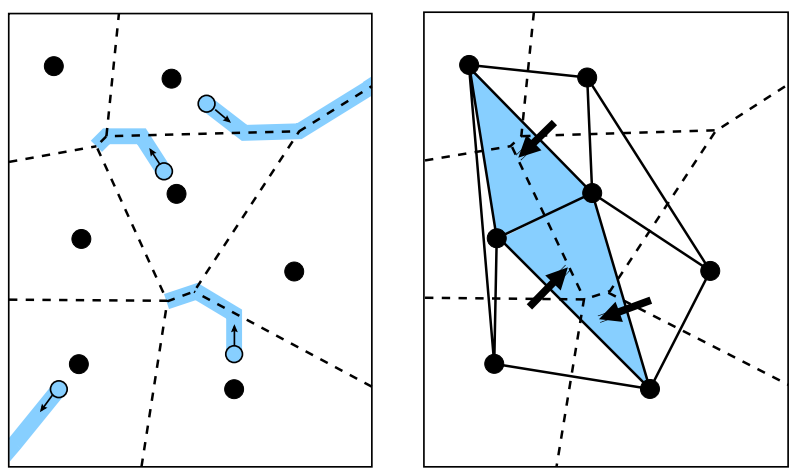

Figure 4: On the left: Some orbits of the flow induced by the points from Figure 2. Note that these orbits are piecewise linear curves. On the right: Triangles containing a maximum are highlighted. An arrow pointing from a triangle $\sigma^{\prime}$ to a triangle $\sigma$ indicates that $\sigma^{\prime}<\sigma$, i.e. $\sigma^{\prime}$ flows into $\sigma$.

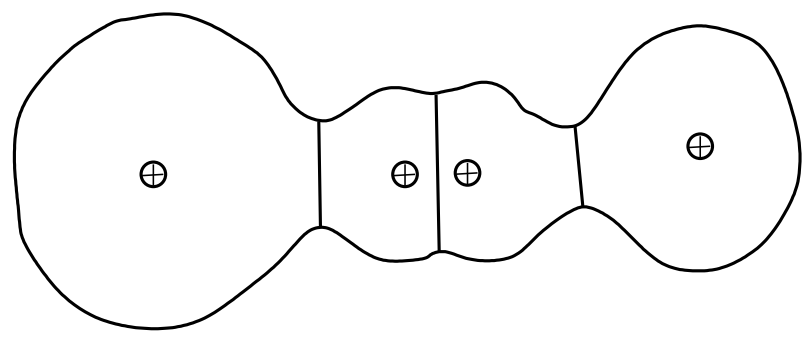

(a)

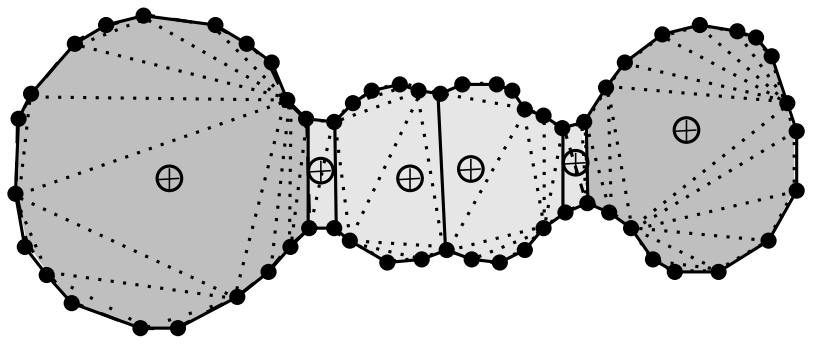

(b)

Figure 5: The closed stable manifolds of the maxima decompose the interior shape bounded by the curve into four segments, the middle two segments are mergeable (a). The discretized version has six segments. The Gabriel edges (solid) among the Delaunay edges (dashed) form the boundaries of these segments. All four middle segments can be merged into a single segment.

Now we turn our attention to $\mathbb{R}^{3}$. In our attempt to compute $F(x)$ for a maximum $x$ in $\mathbb{R}^{3}$, we mimic the setup that we use in $\mathbb{R}^{2}$.

Flow relation in $\mathbb{R}^{3}$. Let $\sigma_{1}, \sigma_{2}$ be two tetrahedra sharing a triangle $t$. We say $\sigma_{1}<\sigma_{2}$ if $\sigma_{1}$ and its dual Voronoi vertex lie on the opposite sides of the plane of $t$. 
It follows from the definition of $<$ that if $\sigma_{1}<\sigma_{2}$, then the radius of the circumsphere of $\sigma_{1}$ is smaller than the radius of the circumsphere of $\sigma_{2}$. Thus, as in $\mathbb{R}^{2}$, the transitive closure $<^{*}$ is acyclic. For a maximum $x$ let

$$
\widetilde{F}(x)=\bigcup_{\sigma^{\prime}<^{*} \sigma} \sigma^{\prime} \text { where } x \in \sigma .
$$

So far everything seems analogous to the two dimensional case, but here we face two difficulties. First, Lemma 3 is no longer valid, i.e. it may be that $F(x) \neq \widetilde{F}(x)$ for a maximum $x$. This is mainly because the stable manifolds of index 2 critical points may not be composed of Delaunay triangles. However, we could use $\widetilde{F}(x)$ as an approximation to $F(x)$. But, we face another difficulty. It might be that $\widetilde{F}(x)$ and $\widetilde{F}\left(x^{\prime}\right)$ are not disjoint for two maxima $x$ and $x^{\prime}$. The reason is that, for a tetrahedron $\sigma$, there may exist more than one tetrahedron $\sigma^{\prime}$ so that $\sigma<\sigma^{\prime}$. This may lead $\sigma$ to flow into two or more different maxima. However, it is interesting to notice the following.

Observation 5 There exist no three tetrahedra $\sigma_{1}, \sigma_{2}, \sigma_{3}$ so that a tetrahedron $\sigma$ satisfies $\sigma<\sigma_{i}$ for $i=1,2,3$.

In order to get pairwise disjoint sets $\widetilde{F}(x)$ we change the relation $<$ to a new relation $\widetilde{<}$ so that for a tetrahedron $\sigma$ there are no two tetrahedra $\sigma_{1}, \sigma_{2}$ with $\sigma \widetilde{<} \sigma_{1}$ and $\sigma \widetilde{<} \sigma_{2}$. Note that the height of a maximum $x$ is its squared distance to the closest sample point in $P$. It is equal to the squared circumradius of the tetrahedron containing $x$. Define the strength of a tetrahedron $\sigma$ as the largest of the heights of all maxima that it flows into.

Strengthened flow relation. We say $\sigma_{1} \widetilde{<} \sigma_{2}$ if

(1) $\sigma_{1}<\sigma_{2}$ and

(2) there is no other tetrahedron $\sigma_{3}$ with $\sigma_{1}<\sigma_{3}$ and the strength of $\sigma_{3}$ is larger than $\sigma_{2}$. In case the strengths of $\sigma_{3}$ and $\sigma_{2}$ are same, we choose $\sigma_{2}$ arbitrarily from $\sigma_{3}$ and $\sigma_{2}$ for the relation.

The transitive closure $\widetilde{<}^{*}$ is acyclic since $<^{*}$ is. Now for a maximum $x$ we redefine $\widetilde{F}(x)$ as

$$
\widetilde{F}(x)=\bigcup_{\sigma^{\prime} \widetilde{<}^{*} \sigma} \sigma^{\prime} \text { where } x \in \sigma .
$$

The sets $\widetilde{F}(x)$ are pairwise disjoint since no tetrahedron can flow into more than one maximum. We compute these sets as an initial segmentation of the shape represented by the finite sample $P$. One can separate the segments for interior and exterior shapes by reconstructing the boundary of the shape from its sample using any of the known surface reconstruction algorithms.

We sort the maxima in decreasing order of their strengths and process them in this order. So, when we process a maximum $x$, all tetrahedra flowing into $x$ and having a strength larger than that containing $x$ have been claimed by some other maxima processed earlier. This is what is required by the definition of $\widetilde{F}(x)$. 


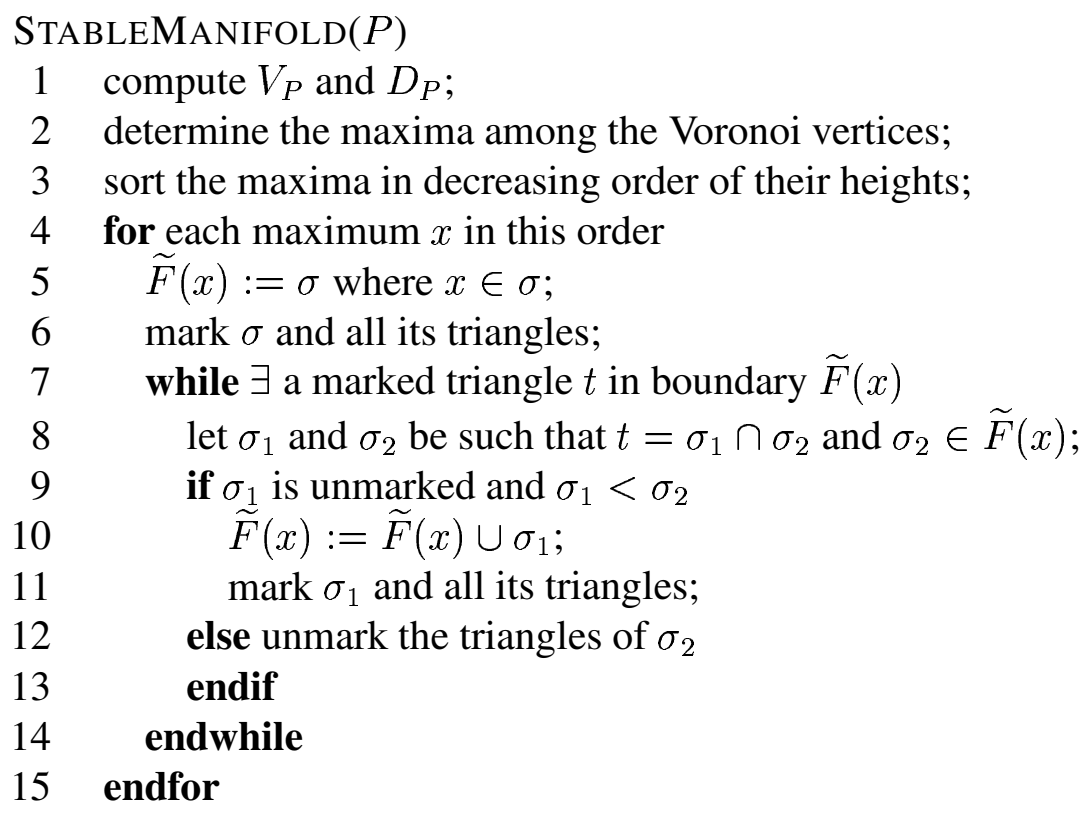

Sometimes closed stable manifolds segment a shape unnecessarily into small features. For example, small perturbations in a shape can cause insignificant segmentation, see for example Figure 5(a). Also, at the discrete level, sampling artifacts may introduce even smaller segments, see Figure 5(b). We propose merging such small segments till two adjacent segments differ significantly.

For a shape let $S(x)$ be a stable manifold of an index $d-1$ critical point $x$ which belongs to the boundary of a closed stable manifold $F(y)$ for a maximum $y$. We say $F(y)$ is $\rho$-shallow with respect to $S(x)$ if as $h(y) \leq \rho h(x)$, i.e., the distances $h(x)$ and $h(y)$ are close to each other measured by a threshold $\rho>1$.

$\rho$-Mergeable stable manifolds. Two closed stable manifolds $F\left(x_{1}\right)$ and $F\left(x_{2}\right)$ for two maxima $x_{1}$ and $x_{2}$ are $\rho$-mergeable if they are $\rho$-shallow with respect to a shared stable manifold on their boundaries. In $\mathbb{R}^{3}$, this means that two stable manifolds of two maxima are $\rho$-mergeable if they are both $\rho$-shallow with respect to a stable manifold of index 2 shared by their boundaries.

Merging all mergeable closed stable manifolds we obtain the feature segmentation of $\Sigma$. For example, the middle two segments for the curve in Figure 5(a) are mergeable. We can translate the definitions and hence the merging algorithm to the discrete setting easily. The distance of a critical point $x$ is measured with the squared circumradius of the lowest dimensional Delaunay simplex that contains $x$. This means, in $\mathbb{R}^{2}$, we merge two closed stable manifolds $F\left(x_{1}\right)$ and $F\left(x_{2}\right)$ if both the triangles containing $x_{1}$ and $x_{2}$ have circumradii less than $\rho$ times the circumradius of the saddle edge shared by $F\left(x_{1}\right)$ and $F\left(x_{2}\right)$.

In $\mathbb{R}^{3}$, we compute approximations $\widetilde{F}(x)$ to a closed stable manifold $F(x)$ for a maximum $x$. Mimicking the definition and the algorithm in $\mathbb{R}^{2}$ we define mergability of two approximated stable manifolds as follows.

$\rho$-mergeable approximate stable manifolds. Let $\widetilde{F}\left(x_{1}\right)$ and $\widetilde{F}\left(x_{2}\right)$ be two approximated stable manifolds that share a triangle $t$. We say $\widetilde{F}\left(x_{1}\right), \widetilde{F}\left(x_{2}\right)$ are $\rho$-mergeable if both the tetrahedra containing $x_{1}$ 
and $x_{2}$ have circumradii less than $\rho$ times the circumradius of $t$.

The final algorithm to compute a feature segmentation of a shape bounded by $\Sigma \subseteq \mathbb{R}^{3}$ from a sample $P$ is described below.

\section{$\operatorname{SEGMENT}(P, \rho)$ \\ 1 Stablemanifold $(P)$; \\ 2 Merge all $\rho$-mergeable segments; \\ 3 Output the resulting decomposition;}

\subsection{Segmentation experiments}

We implemented SEGMENT in C++ using the Delaunay triangulation code of CGAL [43]. SEGMENT produces the decomposition of the interior as well as exterior shape. In order to separate the components of the interior shape $\operatorname{In}(\Sigma)$ from those of the exterior shape $\operatorname{Ex}(\Sigma)$, we used a surface reconstruction program called Tight CoCONE designed by Dey and Goswami [12]. This program designates the Delaunay tetrahedra that approximate $\operatorname{In}(\Sigma)$. The segmentation imposed by SEGMENT on these tetrahedra are shown as output.

Figure 6 shows the dependency of the segmentation on the parameter $\rho$. The leftmost picture shows the segmentation of CACTUS dataset without merging $(\rho<1)$ and the three pictures on the right show the results for $\rho=1.1,1.8,2.0$ respectively.

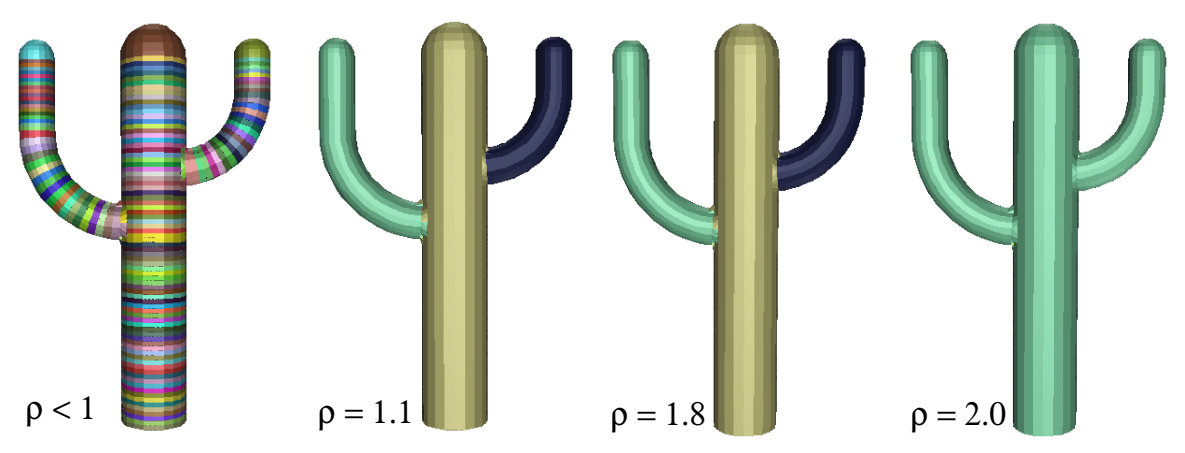

Figure 6: Effect of the merging threshold $\rho$ on segmentation

Figure 7 shows some example segmentations in $\mathbb{R}^{2}$ and $\mathbb{R}^{3}$ respectively. The point samples for the two dimensional models are extracted from the boundary of two dimensional images. As a result they are quite noisy. Nevertheless, the models are segmented nicely. Similarly, we show the segmentation of some three dimensional models some of which are extracted from noisy point samples. For example, the TRICERATOPS and the BULL are segmented nicely even if the corresponding input sample is noisy.

Figure 8 demonstrates the robustness of our method under different samplings of the same surface. The left figure shows the segmentation of the HAND dataset (25626 points) and the right figure shows the segmentation of the decimated version of the same dataset (2301 points).

Figure 9 shows the time taken by the SEGMENT routine together with the interior/exterior separation when run on an $2.8 \mathrm{GHz}$ Pentium IV machine with 1.2GB RAM. 

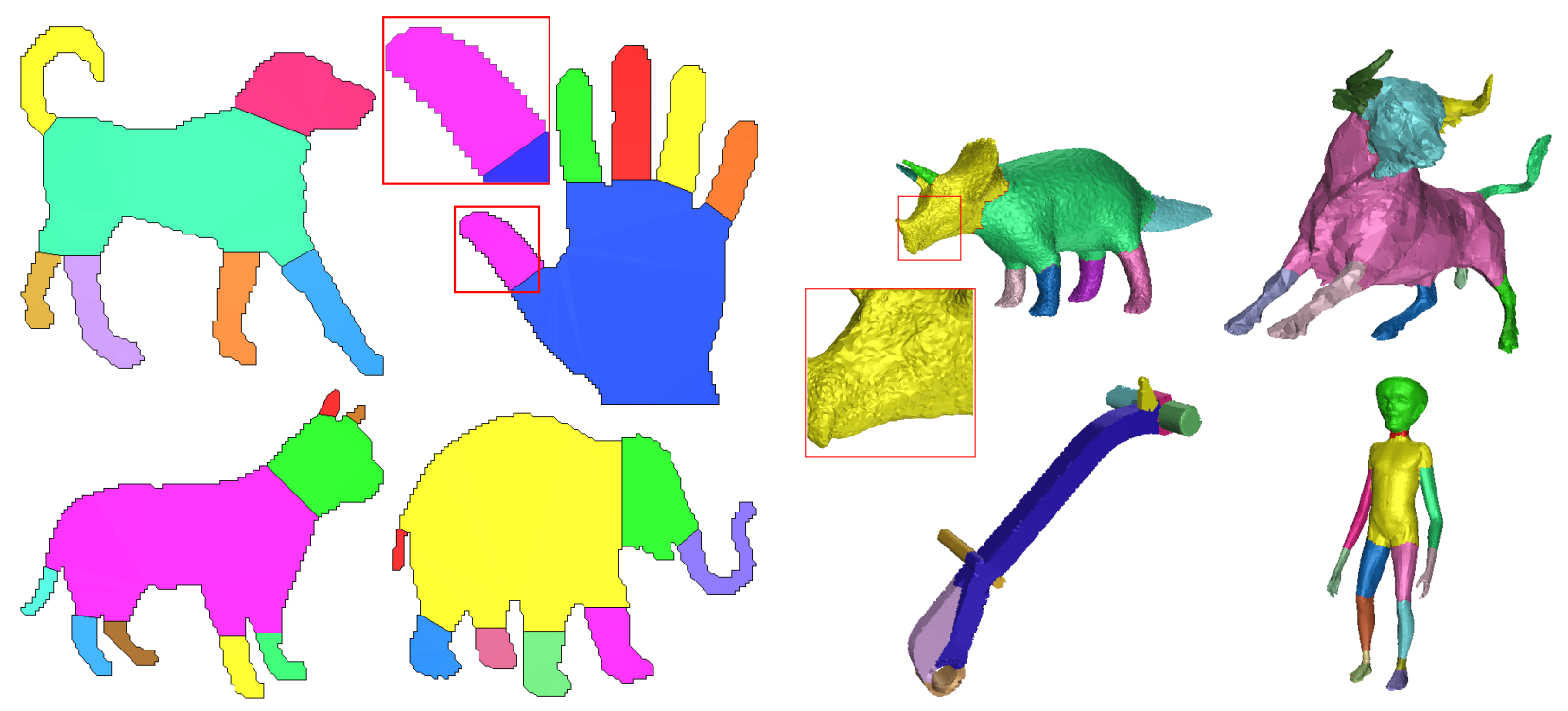

Figure 7: Segmentation of two dimensional and three dimensional models

\section{Matching}

For shape matching we take advantage of our segmentation scheme by matching two shapes with respect to their features. Given a point sample $P$ of a shape boundary $\Sigma$, we identify a small set of significant features from our feature segmentation. These features are then mapped to a set of weighted points called the signature of $\Sigma$. In order to measure the similarity of two shapes, we compare their signatures which boils down to matching two sets of a small number of weighted points.

Signature. Let $R_{P, \Sigma}$ denote the set of features for $\operatorname{In}(\Sigma)$ that the function SEGMENT computes together with Tight Cocone from a point sample $P$ of $\Sigma$. To simplify notations we use $R_{\Sigma}$ for $R_{P, \Sigma}$. By definition a feature $r \in R_{\Sigma}$ is a collection of Delaunay triangles if $\Sigma$ is a curve in two dimensions and it is a collection of Delaunay tetrahedra if $\Sigma$ is a surface in three dimensions. For a Delaunay simplex $\sigma$ let $c_{\sigma}$ and $v_{\sigma}$ denote the centroid and volume of $\sigma$, respectively. The representative point $r^{*}$ and its weight $\hat{r}$ for a feature $r$ are defined as

$$
\begin{aligned}
\hat{r} & =\Sigma_{\sigma \in r} v_{\sigma} \\
r^{*} & =\frac{\Sigma_{\sigma \in r}\left(c_{\sigma} \cdot v_{\sigma}\right)}{\hat{r}} .
\end{aligned}
$$

That is, the weight of $r$ is its volume and its representative point is the weighted average of the centroids of all $\sigma \in r$, weight being the volume of each simplex. We call a feature $r$ significant if its volume is more than a certain fraction of the total volume of the shape. Given a segmentation $R_{\Sigma}$ of the interior shape $\operatorname{In}(\Sigma)$, the signature $\operatorname{sig}(\Sigma)$ is defined as the set of weighted representative points, i.e.,

$$
\operatorname{sig}(\Sigma)=\left\{\left(r^{*}, \hat{r}\right) \mid r \in R_{\Sigma} \text { is significant }\right\} .
$$




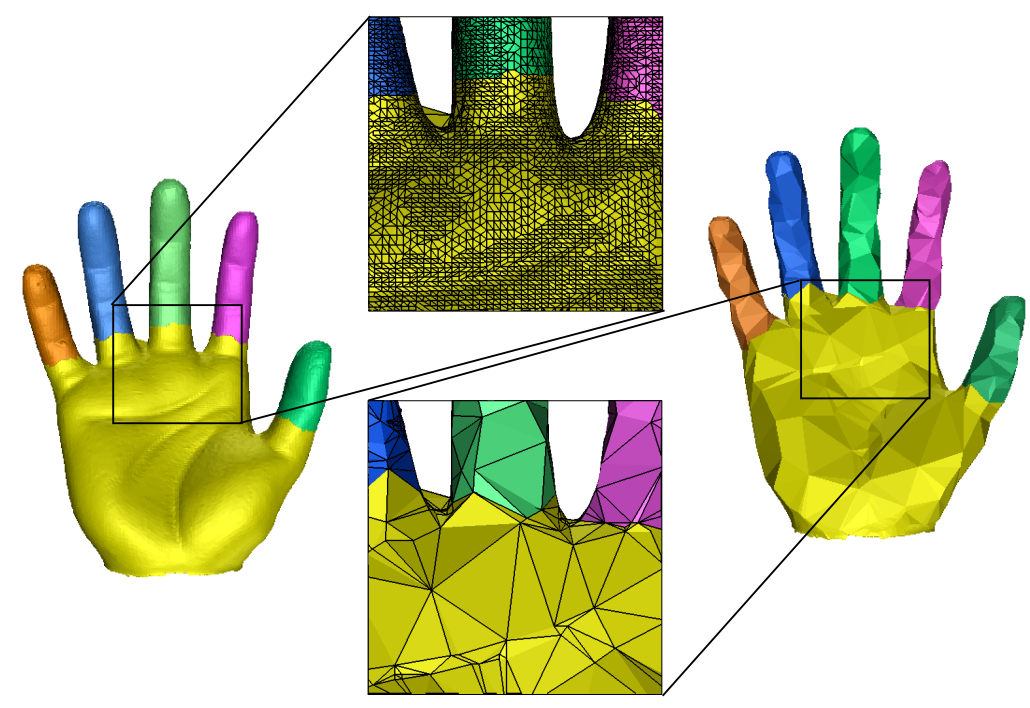

Figure 8: Robustness of the algorithm against varying sampling density

\begin{tabular}{|c|c|c|c|c|}
\hline MODEL & $\begin{array}{c}\text { Num. of } \\
\text { points }\end{array}$ & $\begin{array}{c}\text { Del. } \\
\text { (sec.) }\end{array}$ & $\begin{array}{c}\text { Seg. } \\
(\text { sec. })\end{array}$ & $\begin{array}{c}\text { In/Ex Sep. } \\
\text { (sec.) }\end{array}$ \\
\hline PIG & $37 \mathrm{~K}$ & 15.68 & 19.13 & 14.84 \\
\hline HORSE & $48 \mathrm{~K}$ & 17.89 & 26.79 & 25.14 \\
\hline BOY & $95 \mathrm{~K}$ & 52.71 & 46.3 & 46.02 \\
\hline ALIEN & $120 \mathrm{~K}$ & 70.49 & 56.09 & 54.19 \\
\hline
\end{tabular}

Figure 9: Time for different stages of SEGMENT together with the interior/exterior separation

The amount of similarity between two shapes is measured by first scaling them with bounding boxes and then scoring the similarity between their signatures. In order to score the similarity between two signatures $\operatorname{sig}\left(\Sigma_{1}\right)$ and $\operatorname{sig}\left(\Sigma_{2}\right)$, we need to align them first.

Let $r^{*}, s^{*}$ be the representative points with maximum weights among all representative points in $\operatorname{sig}\left(\Sigma_{1}\right)$ and $\operatorname{sig}\left(\Sigma_{2}\right)$, respectively. We first translate $\operatorname{sig}\left(\Sigma_{2}\right)$ so that $r^{*}, s^{*}$ coincide. Then an alignment is obtained by rotating $\operatorname{sig}\left(\Sigma_{2}\right)$ so that a line segment between $s^{*}$ and another point of $\operatorname{sig}\left(\Sigma_{2}\right)$ aligns with a line segment between $r^{*}$ and another point in $\operatorname{sig}\left(\Sigma_{1}\right)$. Certainly, there are $\Theta(m n)$ alignments possible where $\left|\operatorname{sig}\left(\Sigma_{1}\right)\right|=m$ and $\left|\operatorname{sig}\left(\Sigma_{2}\right)\right|=n$. Since $m, n$ are typically small (less than ten), checking all alignments is not prohibitive.

For each alignment we compute a score based on the matching of weighted points. Both a similarity measure (positive) and a dissimilarity measure (negative) are taken into account while computing the score. The maximum of all the scores is taken to be the amount of similarity and corresponding transformations give the best alignment.

Before we compute the score, the weights of the segments are normalized so that each weight is between 0 and 1. Next, for each point $q^{*} \in \operatorname{sig}\left(\Sigma_{2}\right)$, we determine the Euclidean nearest neighbor, say $p^{*}$, in $\operatorname{sig}\left(\Sigma_{1}\right)$. If $\left\|p^{*}-q^{*}\right\|$ is less than a "threshold", we compute a similarity score as 


$$
1-\left|\frac{\hat{p}-\hat{q}}{\hat{p}+\hat{q}}\right|
$$

where the "threshold" is a parameter that tells how much tolerance we can have for the proximity of two features. The points in $\operatorname{sig}\left(\Sigma_{1}\right)$ and $\operatorname{sig}\left(\Sigma_{2}\right)$ that do not have nearest neighbors in the other set within "threshold" distance contribute to a dissimilarity score which is equal to the negative of their weights. Finally, we add both similarity and dissimilarity scores to obtain the score of matching between the two shapes bounded by $\Sigma_{1}$ and $\Sigma_{2}$.

\subsection{Matching experiments}

We performed the matching experiments both in two and three dimensions. In two dimensions, we created a database of around 350 shapes and in three dimensions the database consisted of signatures of more than 300 point cloud data collected from different web-sites. Some example matching results both in two and three dimensions are shown in Fig 10. Matching of a query shape over the entire database took less than a second. Fig 11(a) shows the similarity matrix of our method on approximately 250 two dimensional shapes divided into 16 categories. Fig 11(b) shows the performance of this method on approximately 200 three dimensional shapes divided into 17 categories. In the similarity matrix the $i$-th row contains the gray scale value corresponding to the 5 best matches in the database for the $i$-th shape as the query.

We find it difficult to compare our technique with other matching algorithms as all of them assume a surface mesh as an input. One novelty of our algorithm is that we do not build any extra data structure other than the Delaunay triangulation of the input point set. Also, there is no costly computation such as approximating geodesic distances as by Hilaga et al. [20]. The shape distribution method of Osada et al. [30] assumes an input mesh. For comparisons we adapted it to point samples as follows. We use the D2 metric, that is, we compute distances between pairs of randomly selected points. Random selection took care of the point density to have a fair comparison. This technique is quite effective for most of the models, however sometimes it creates some anomalies. Figure 12 shows one such example for which our method works properly.

\section{Discussions and conclusions}

Our results have shown that the segmentation is quite robust against small variations in shapes. For example, the human bodies in the first row of the matching table in Figure 10 are segmented similarly, namely into head, torso, two hands and two legs. This is why they matched against the query shape with higher scores than other shapes in the database. The tolerance against shape variations can be attributed to the fact that our approach emphasizes topology more than local geometry. Topological features in terms of the height function change relatively less with the local changes in geometry. There are a number of open questions that remain to be addressed.

In some cases the segmented features deviate visibly from the intuitive ones. For example, the fingers in the hands of Figure 7 and Figure 8 do not get separated from the palm where they meet. A small stump remains attached to the palm for each finger. We believe that we need a refined merging strategy to tackle this problem. 

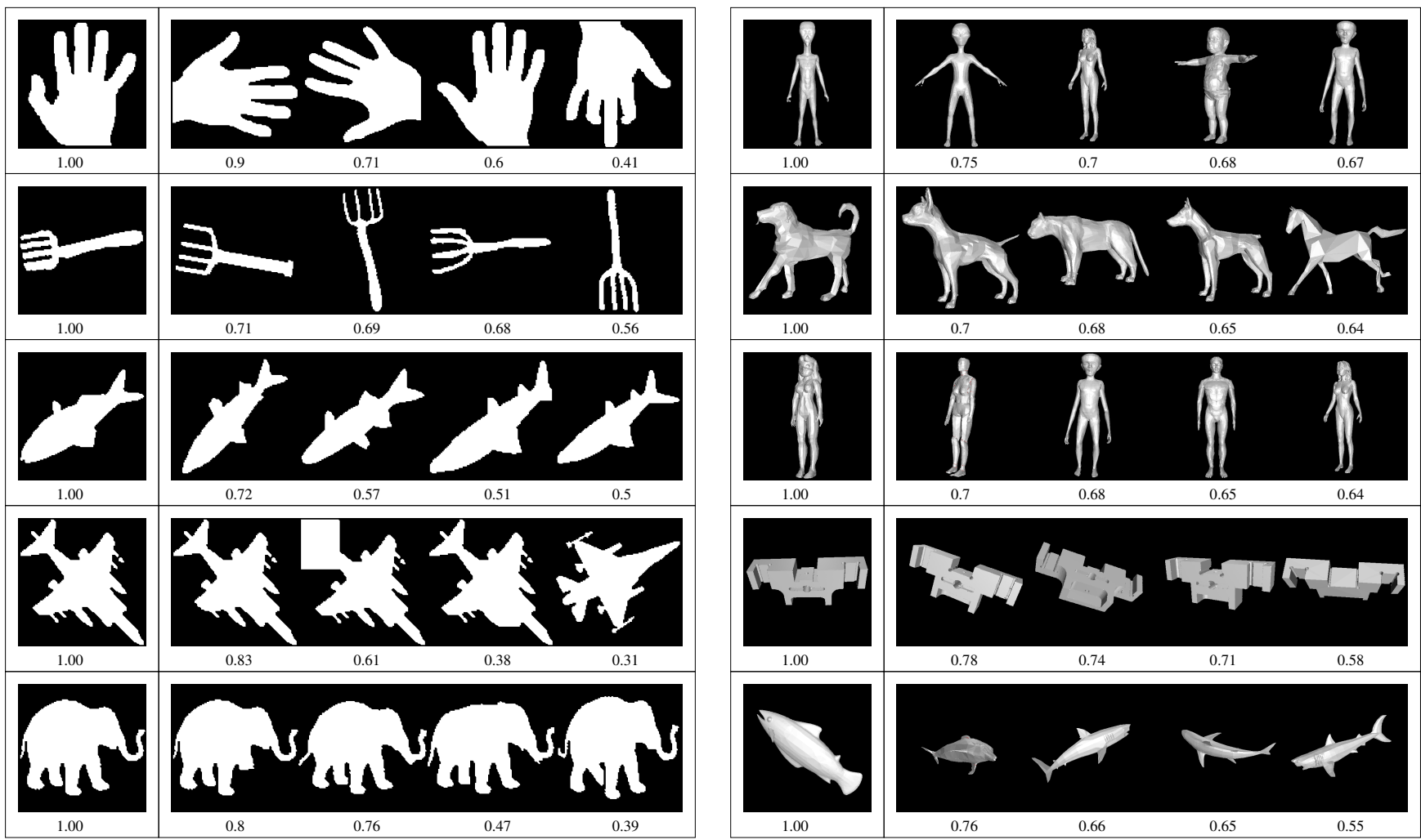

1.00

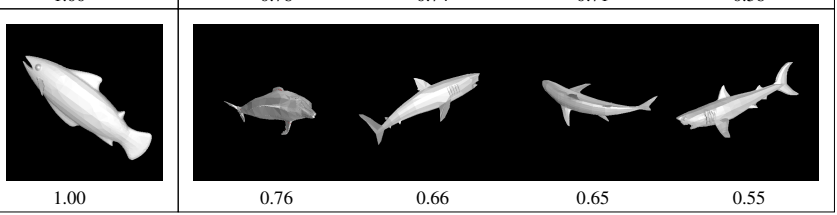

Figure 10: Matching results in two dimensions (left) and three dimensions (right): Models with 5 best scores for each query are shown in a row.

Although we mimicked the definition of features from the continuous space to the discrete domain, there is no quantitative estimate of the approximation. Specifically, can we claim that if the sampling density is beyond a threshold, then all defined features in the shape are approximated well?

Our experiments show that the segmentation algorithm is quite robust against reasonable amount of noise. In presence of noise, it becomes more difficult to separate the segments decomposing the interior shape from the segments decomposing the exterior one. Elsewhere, we showed that a robust surface reconstruction method [13] that can handle noisy point sample is helpful in these situations [14]. More thorough investigations are needed to handle noise.

In the shape matching algorithm we used the volumes of the segments as the weight of the representative points. In a sense, we took the volume of a feature to be its signature. Are there other measures that capture the signature of a feature more effectively? Currently we are investigating all these questions.

\section{Acknowledgements.}

The first and third authors acknowledge the support of the NSF grants DMS-0138456 and DMS-0310642 and an ARO grant DAAD19-02-0347. The second author acknowledges the support of IST(ECG) programme under contract no. IST-2000-26473 and a start-up grant from the Ohio State University. 


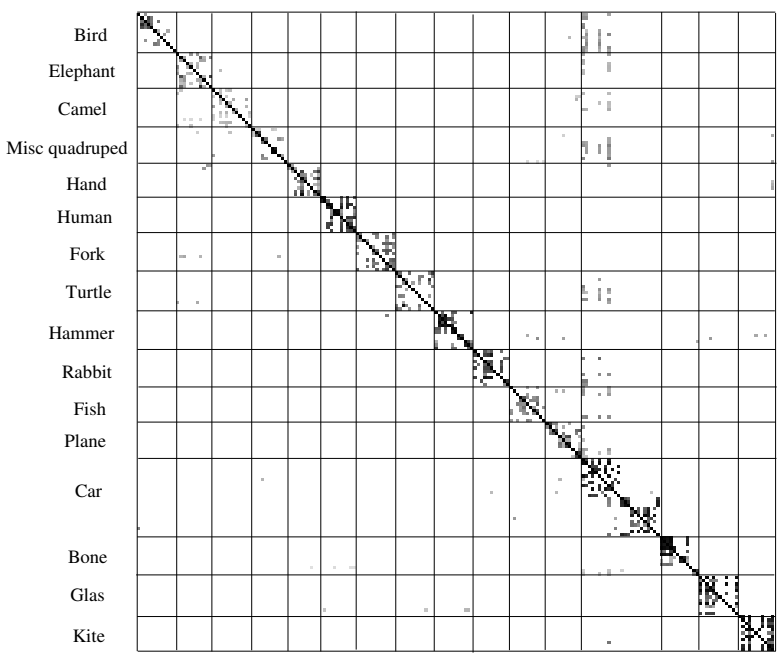

(a)

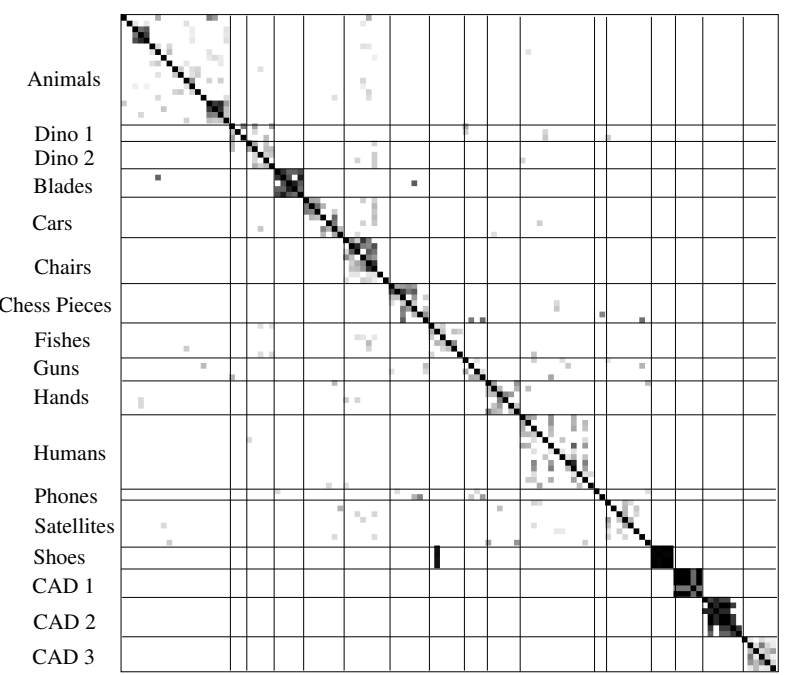

(b)

Figure 11: Similarity Matrix for two dimensional (left) and three dimensional (right) shapes.

\section{References}

[1] H. Alt and L. J. Guibas. Discrete geometric shapes: matching, interpolation, and approximation: a survey. Tech. report B 96-11, EVL-1996-142, Institute of Computer Science, Freie Universität Berlin, 1996.

[2] K. Arbter, W. E. Snyder, H. Burkhardt and G. Hirzinger. Application of affine-invariant fourier descriptors to recognition of 3-d objects. IEEE Trans. Pattern Analysis Machine Intelligence. 12 (1990), 640-647.

[3] E. M. Arkin, L. P. Chew, D. P. Huttenlocher, K. Kedem and J. S. Mitchell. An efficiently computable metric for comparing polygonal shapes. IEEE Trans. PAMI 13 (1991), 209-216.

[4] A. P. Askbrook, N. A. Thacker, P. I. Rockett and C. I. Brown. Robust recognition of scaled shapes using pairwise geometric histograms. BMVC (1995), 503-512.

[5] R. Basri, L. Costa, D. Geiger and D. Jacobs. Determining the similarity of deformable shapes. Vision Research 38 (1998), 2365-2385.

[6] S. Belongie and J. Malik. Matching with shape contexts. IEEE Trans. PAMI 24 (2002), 509-522.

[7] P. Besl and R. C. Jain. Three-dimensional object recognition. Comput. Surveys 17 (1985), 75-145.

[8] P. J. Besl and N. D. McKay. A method for registration of 3-D shapes. IEEE Trans. PAMI 14 (1992) 239-256.

[9] T. A. Cass. Robust Affine Structure Matching for 3D Object Recognition. IEEE Trans. PAMI 20 (1998), 1265-1264.

[10] Y. Chen and G. Medioni. Object Modelling by Registration of Multiple Range Images. Image and Vision Computing 10 (1992) 145-155.

[11] L. P. Chew, M. T. Goodrich, D. P. Huttenlocher, K. Kedem, J. M. Kleinberg and D. Kravets. Geometric Pattern Matching under Euclidean motion. Comput. Geom. Theory Applications 7 (1997) 113-124.

[12] T. K. Dey and S. Goswami. Tight Cocone : A water-tight surface reconstructor. J. Computing Infor. Sci. Engin. 30 (2003), 302-307. 


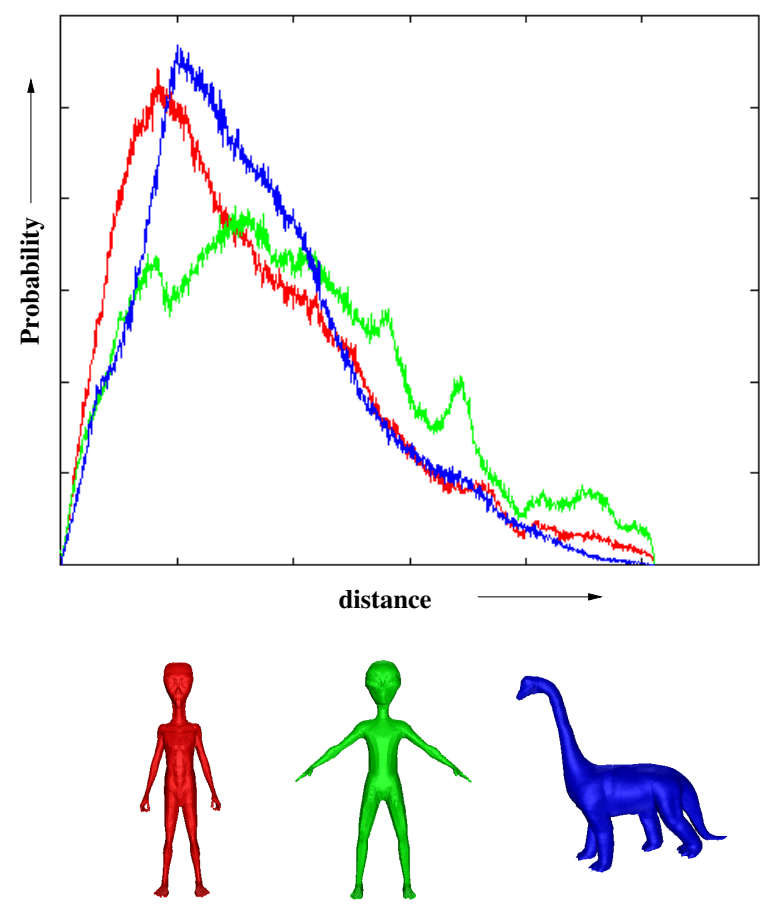

Figure 12: In the shape distribution method the probability distribution plot of D2 distances shows a better match between the left alien and the dinosaur. Our method matches the two aliens better than with the dinosaur.

[13] T. K. Dey and S. Goswami. Provable surface reconstruction from noisy samples. Proc. 20th Annu. Sympos. Comput. Geom. (2004), 330-339.

[14] T. K. Dey, J. Giesen and S. Goswami. Shape segmentation and matching from noisy point clouds. Proc. Eurographics Sympos. Point-Based Graphics (2004), 193-199.

[15] H. Edelsbrunner. Surface reconstruction by wrapping finite point sets in space. Ricky Pollack and Eli Goodman Festschrift, eds. B. Aronov, S. Basu, J. Pach and M. Sharir, Springer-Verlag, 379-404.

[16] H. Edelsbrunner, D. Letscher and A. Zomorodian. Topological persistence and simplification. Discrete Comput. Geom. 28 (2002), 511-533.

[17] H. Edelsbrunner, M. A. Facello and J. Liang. On the definition and the construction of pockets in macromolecules. Discrete Apl. Math. 88 (1998), 83-102.

[18] J. Giesen and M. John. The flow complex: a data structure for geometric modeling. Proc. 14th Ann. ACM-SIAM Sympos. Discrete Algorithms (2002), 285-294.

[19] S. K. Gupta, W. C. Regli and D. S. Nau. Manufacturing feature instance: which ones to recognize? Proc. Sympos. Solid Modeling (1995), 141-152.

[20] M. Hilaga, Y. Shinagawa, T. Komura and T. Kunni. Topology matching for fully automatic similarity estimation of 3D shapes. Proc. SIGGRAPH 2001 (2001), 203-212. 
[21] D.P.Huttelnocher, G.A.Klanderman and W.J.Rucklidge. Computing images using the Hausdorff distance. IEEE Trans. PAMI 15 (1993), 850-863.

[22] C. E. Jacobs, A. Finkelsten and D. H. Salesin. Fast multiresolution image querying. Proc. SIGGRAPH '95, 277-286.

[23] A. E. Johnson and M. Hebert. Using spin-images for efficient multiple model recognition in cluttered 3-d scenes. IEEE Trans. PAMI 21 (1999), 433-449.

[24] S. Katz and A. Tal. Hierarchical mesh decomposition using fuzzy clustering and cuts. SIGGRAPH 2003, ACM Trans. Graphics 22 (2003), 954-961.

[25] M. Kazhdan, T. Funkhouser and S. Rusinkiewicz. Rotation invariant spherical harmonic representation of 3D shape descriptors. Sympos. Geom. Process. (2003).

[26] F. Leymarie and B. Kimia. The shock scaffold for representing 3D shape. Proc. 4th Internat. Workshop Visual Form. (2001), LNCS 2059, Springer-Verlag, 216-229.

[27] X. Li, T. W. Toon, T. S. Tan and Z. Huang. Decomposing polygon meshes for interactive applications. Proc. Sympos. Interactive 3D Graphics (2001), 35-42.

[28] S. Loncaric. A survey of shape analysis techniques. Pattern Recognition 31 (1998) 983-1001.

[29] A. P. Mangan and R. T. Whitaker. Partitioning 3D surface meshes using watershed segmentation. IEEE Trans. Visualization Comput. Graphics 5 (1999), 308-321.

[30] R. Osada, T. Funkhouser, B. Chazelle and D. Dobkin. Matching 3D Models with Shape Distribution. Proc. Shape Modelling Internat. (2001).

[31] K. Grove. Critical point theory for distance functions. Proc. Sympos. Pure Math. 54 (1993), 357-385.

[32] R. J. Prokop and A. P. Reeves. A survey of moment based techniques for unoccluded object representation and recognition. CVGIP:Graphics Models and Image Processing 54 (1992), 438-460.

[33] Y. Rubner, C. Tomasi and L. Guibas. A metric for distributions with applications to image databases. 6th ICCV (1998), 59-66.

[34] S. Sclaroff and A. P. Pentland. Modal Matching for Correspondence and Recognition. IEEE Trans. PAMI 17 (1995) $545-561$.

[35] T. B. Sebastian, P. N. Klein and B. Kimia. Recognition of shapes by editing shock graphs. Proc. ICCV (2001), 755-762.

[36] J. A. Sethian. Level set methods and fast marching methods: evolving interfaces in computational geometry, fluid mechanics, computer vision, and materials science. Cambridge University Press, 2000.

[37] K. Siddiqi, A. Shokoufandeh, S. J. Dickinson and S. W. Zucker. Shock graphs and shape matching. Computer Vision (1998), 222-229.

[38] R. Sonthi, G. Kunjur and R. Gadh. Shape feature determination using the curvature region representation. Proc. Sympos. Solid Modeling (1997), 285-296.

[39] G. Taubin and D. Cooper. Geometric invariance in computer vision, Chapter Object Recognition Based on Moment (of algebraic) Invariants, MIT press, 1992.

[40] R. C. Veltkamp and M. Hagedoorn. State-of-the-art in shape matching. Technical report UU-CS-1999-27, Utrecht University, the Netherlands, 1999.

[41] I. Weiss and M. Ray. Model-based recognition of 3D object from single vision. IEEE Trans. PAMI 23 (2001), 116-128. 
[42] D. Zhang and M. Hebert. Harmonic maps and their applications in surface matching. CVPR '99 (1999).

[43] http://www.cgal.org 